PSYCHOMETRIKA-VOL.48, No.3, SEPTEMBER,1983

http://www.psychometrika.org/

\title{
A NOTE ON THE USE OF DIRECTIONAL STATISTICS IN WEIGHTED EUCLIDEAN DISTANCES MULTIDIMENSIONAL SCALING MODELS
}

\author{
CHARLES L. JONES \\ MCMASTER UNIVERSITY AND UNIVERSITY OF TORONTO
}

Keywords:multidimensional scaling, directional data.

Reprint requests should be addressed to Charles L. Jones, Sociology Department, 563

Spadina Avenue, University of Toronto, Toronto, Ontario, M5S IAI, Canada.

Material support for work referred to herein was received from SSRC grant HR1883/1, SSHRC grant 410-770860, and from McMaster University.

Thanks are due to A. P. M. Coxon, M. J. Prentice, M. A. Stephens and an anonymous reviewer.

0033-3123/83/0900-5027 @1983 The Psychometric Society 


\title{
A NOTE ON THE USE OF DIRECTIONAL STATISTICS IN WEIGHTED EUCLIDEAN DISTANCES MULTIDIMENSIONAL SCALING MODELS
}

\author{
CHARLES L. JONES \\ MCMASTER UNIVERSITY AND UNIVERSITY OF TORONTO
}

\begin{abstract}
The weighted euclidean distances model in multidimensional scaling (WMDS) represents individual differences as dimension saliences which can be interpreted as the orientations of vectors in a subject space. It has recently been suggested that the statistics of directions would be appropriate for carrying out tests of location with such data. The nature of the directional representation in WMDS is reviewed and it is argued that since dimension saliences are almost always positive, the directional representations will usually be confined to the positive orthant. Conventional statistical techniques are appropriate to angular representations of the individual differences which will yield angles in the interval $(0,90)$ so long as dimension saliences are nonnegative, a restriction which can be imposed. Ordinary statistical methods are also appropriate with several linear indices which can be derived from WMDS results. Directional statistics may be applied more fruitfully to vector representations of preferences.
\end{abstract}

Key words: multidimensional scaling, directional data.

The weighted euclidean distances model in multidimensional scaling (MDS) is due to Bloxom [Note 1], Horan [1969], and Carroll and Chang [1970]. Schiffman, Reynolds and Young [1981] attempt to standardise terminology by calling it the weighted multidimensional scaling (WMDS) model. Each individual is characterised by a weight on each of the dimensions of a common stimulus matrix. Various estimation methods have been used, but in all cases the solution is obtained conditional upon the adoption of certain reasonable but arbitrary normalizing conventions. Carroll and Chang [1970] pointed out that interpretations of the differential salience of dimensions for the subjects should not be based on the absolute sizes of the dimension weights, but rather on their relative sizes, within each subject, [see McCallum, 1977]. This interpretation lends itself to a graphical representation of individual subjects as vectors (in a "subject space") whose orientations reflect their patterns of weights which in turn represent the differential saliences of the dimensions.

In Ramsay's MULTISCALE version of WMDS, the weights for any subject are normalized so that they sum to the number of dimensions. However, in INDSCAL, S-INDSCAL and ALSCAL versions of WMDS, the weights for any subject are estimated in a way that constrains their sum of squares to be (approximately) the proportion of variation in the subject's data, (or some transformation of those data) that is accounted for by the model. When attention is focused on the relative sizes of weights within subjects, it has often been found convenient to set aside this information about goodness of fit, and to re-normalize the weights so that their sum of squares is 1.00 for each subject.

Once the subject vectors are normalized they can be represented as points on the circumference of a circle (for a two-dimensional analysis) or on the surface of a sphere (for three dimensions), or on a hypersphere (for a higher number of dimensions). 
Schiffman et al. [1981J have emphasised directional interpretations of individual differences in the WMDS model and have drawn attention to a class of statistical procedures appropriate for directions, [Mardia, 1972; Batschelet, 1981]. They advocate the use of a multi-sample test for location, due to Watson and Williams [1956J and which they call ANAVA (analysis of angular variation). Schiffman et al. discuss some of the assumptions of this test and note (pp. 307-308) that the subject weights from the WMDS model are only conditionally independent from one subject to another, since the choice of different stimulus configurations would imply different sets of subject weights. Even a rotation of the stimulus configuration will generate a different set of subject weights. The appropriate reaction to this problem may be to err on the side of caution when applying the Watson-Williams test, (as indeed is suggested by Schiffman et at). However, we shall see that there is no reason to use directional statistics when carrying out statistical tests on subject weights from the WMDS model. The observations will still be only conditionally independent, but the more widely understood linear (as opposed to directional) statistics can be used.

Under the null hypothesis, the Watson-Williams test (Schiffman et al.'s ANAVA) assumes that the directional observations are sampled from a population distributed according to the r-dimensional Von Mises distribution. This distribution is symmetrical about a polar axis attaining maximum density at the pole and minimum density at the anti-pole. In two dimensions it is known as the Von Mises distribution and in three dimensions as the Fisher distribution. As Schiffman et al. point out, the individual weights are almost always estimated as being positive, and some computer programmes allow the option of restricting them to being nonnegative. It may be added that negative subject weights have no sensible interpretation in the WMDS model. The fact that weights are almost always positive has the effect of truncating the distribution of corresponding directions. Schiffman et al. [1981, p. 304J note that subject vectors are distributed, "... with low probability in the nonpositive orthants (quadrants, octants etc.)", but this way of phrasing the matter hardly does justice to the smallness of this "low probability". Furthermore, they ignore the fact that the nonpositive orthants constitute an increasingly large proportion of the surface of a hypersphere as the dimensionality increases. This proportion is given as $\left(2^{r}-1\right) / 2^{r}$ where $r$ is the number of dimensions considered. Given the degree of truncation, it seems unlikely that the distributional assumptions of the Watson-Williams test are met when the test is applied to directional data from the WMDS model. Nonparametric tests for directional data are available [Wheeler \& Watson, 1964J but are appropriate only for the two-dimensional case.

The problem of correct treatment of directional observations which are restricted to the interval $(0,180)$ was discussed by Batschelet $[1975 \mathrm{~J}$, who concluded that the best plan is to treat such data (in the form of angles measured in degrees or in radians) as if they were linear. Angular data in the positive orthant will be on the interval $(0,90)$. In a two dimensional solution the pattern of subject weights (though not the explained variation) is given as a single angle for each subject. In three dimensions two angles are necessary to capture the pattern of dimension saliences. Since at least two computer programmes allow the WMDS model to be estimated with the constraint that subject weights be nonnegative, researchers can make sure that angular data representing individual differences in this model will always be in the positive orthant.

The orientation of an individual subject vector is obtained through standard trigonometry by taking the inverse tangents of the ratios of weights for that subject, (assuming orthogonal axes of the subject space). Coxon and Jones [1974, 1978J reported linear (as opposed to directional) statistical analyses of the logarithms of within-subject ratios of weights. This was similar to the approach advocated by Batschelet, the only 
difference being his use of the inverse tangent transformation, rather than the logarithm. Neither transformation would affect the rank order of the ratios of weights.

Other ways to look at the WMDS subject weights have been suggested. Young and Lewyckyj [Note 2] describe how sine-based or tangent-based transformations of the angular representation of subject weights could result in "relative subject weight index" variables, ranging between plus minus 1.00 , with a zero value meaning that the subject weights a given pair of dimensions just as the typical subject does, a positive value indicating a tendency (relative to the average subject) to overemphasize one of the pair of dimensions, and a negative value indicating the reverse tendency. Examples of this method are given by Young and Lewyckyj, by Schiffman et al. (pp. 175-176), and by Coxon and Jones [1980] who use it to display subject weights estimated by MULTI SCALE. Young [Note 3] has introduced a related measure (the "weirdness index") of how far an individual's weights depart from the pattern of the typical subject. Each of these three measures could be used with linear statistical methods.

Schiffman et al. give another interpretation of WMDS weights which they call "flattened weights". Again dealing with subject vectors which have been normalized (sum of squares 1.00), it can be shown that the sum (over $r$ dimensions) of cross products of weights estimated for any two subjects is the cosine of the angle between the two weight vectors, and that this can be expressed as an arc-distance between points representing the ends of the subject vectors on an r-dimensional hypersphere. Such arc distances are monotone with euclidean distances in a space with one less dimension, and a nonmetric MDS method can therefore "flatten" the directional representation of the subject weights into a space with $r-1$ dimensions. The subjects weights from WMDS are then considered as a data matrix with $r-/$ columns and as many rows as there are subjects. Standard linear statistical methods may be used with such data if it is borne in mind that the observations are not independent of one another. Another possibility might be to use the analysis of variance for proximities data, recently developed by Hubert, Golledge, and Costanzo [1982]. As before, it must be remembered that the WMDS weights are not independent of one another, unless an externally supplied group space is used in estimation procedure. Even then, the weights, though independent of one another are conditional on that particular group space.

Young [Note 3J has recently suggested a different "flattening" transformation of WMDS weights, and incorporated it into his ALSCAL program.

\footnotetext{
"The ALSCAL-82 transformation involves normalizing each subject's weights so that their sum is 1.00. This defines a set of $r$ 'variables', whose elements are the flattened weights. The roth variable is dropped and the remaining variables are centred and normalized. This is the complete definition of the flattening transformation used in ALSCAL-82". (p. 9).
}

This representation of subject weights is reminiscent of Ramsay's MULTISCALE, where the weights for any individual sum to the number of dimensions. Note that it is the sum, not the sum of squares that is used as a normalizing factor, and that this is only sensible if all weights are positive, (plausible in the WMDS model, but not likely in the vector model for the analysis of preferences). Dropping the last "variable" removes a linear dependency in the set of "flattened weights", which can then be used in linear statistical procedures, (though with the usual caveats about the weights being conditional on a group space, and not necessarily independent of one another). Thus both Young and Coxon and Jones recommend linear procedures, though they use different transformations.

It is worth repeating that all the approaches discussed above re-normalize the individual weight vectors in some way, and thereby give equal emphasis to all individuals, including those whose data are very poorly fit by the model, (a not uncommon occurrence with the WMDS model). Those who use WMDS subject weights as summary measures of 
individual characteristics are therefore advised to include some index of how well each subject's data are explained by the model as a control variable, and weight the analysis in terms of this variable, or remove its linear effects before interpreting an association between WMDS weights and some externally defined characteristic of the subjects.

The vector model for preferences judgments represents individual differences as angular differences between subject vectors. Since this directional information is not necessarily restricted to the positive orthant, (as is usually the case with the WMDS model), the directional statistics discussed by Schiffman et al. may usefully be applied to such data. A complete example is given in Coxon and Jones [1978], and the matter is further discussed in Coxon and Jones [1979], and in Jones [in press].

\section{REFERENCE NOTES}

1. Bloxom, B. Individual differences in multidimensional scaling. Research Bulletin 68-45. Princeton, N.J.: Educational Testing Service, 1968.

2. Young, F. W. \& R. Lewyckyj. ALSCAL-4 User's Guide. 2nd edition. Psychometric Laboratory. University of North Carolina: Chapel Hill, NC 27514.1979.

3. Young, F. W. Enhancements in ALSCAL-82. Proceedings of the Seventh Annual SAS Users Group, Cary, NC: The SAS Institute. 1982. pp. 633-642.

\section{REFERENCES}

Batschelet, E., Contribution to the discussion of a paper by K. V. Mardia. Journal of the Royal Statistical Society Series B, 1975,37,378.

Batschelet, E., Circular statistics in biology. New York: Academic Press, 1981.

Carroll, J. D. \& Chang, J. J., Analysis of individual differences in multidimensional scaling via an N-way generalization of "Eckart-Young" decomposition. Psychometrika, 1970, 35, 238-319.

Coxon, A. P. M. \& Jones, C. L., Occupational similarities: subjective aspects of social stratification. Quality and Quantity, 1974,8,139-157.

Coxon, A. P. M. \& Jones, C. L. The images ofoccupational prestige. London: Macmillan, 1978.

Coxon, A. P. M. \& Jones, C. L., Measurement and meanings. London: Macmillan, 1979.

Coxon, A. P. M. \& Jones, C. L. Multidimensional scaling: exploration to confirmation. Quality and Quantity, 1980,14(1),31-73.

Horan, C. B. Multidimensional scaling: combining observations when individuals have different perceptual structures. Psychometrika, 1969,34, 139-165.

Hubert, L. J., Golledge, R. G., \& Costanzo, C. M. Analysis of variance procedures based on a proximity measure between subjects. Psychological Bulletin, 1982,91, (2),424-430.

Jones, C. L. Analysis of preferences as directional data. Quality and Quantity (in press).

Mardia, K. V. Statistics ofdirectional data. New York: Academic Press, 1972.

McCallum, R. C. Effects of conditionality on INDSCAL and ALSCAL weights. Psychometrika, 1977 42, (2), 297-305.

Schiffman, S. S., Reynolds, M. A. \& Young, F. W.lntroduction to multidimensional scaling. New York: Academic Press, 1981.

Watson, G. S., \& Williams, E. J. On the construction of significance tests on the circle and the sphere. Biometrika, 1956,43,344-352.

Wheeler, S. \& Watson, G. S. A distribution-free two sample test on a circle. Biometrika, 1964,51,256-257. 